\title{
Flexible three-dimensional microelectrode array for neural applications
}

\author{
A.C. Peixoto $^{1 *}$, S.B. Goncalves ${ }^{1}$, F.Pinho ${ }^{1}$, A.F.Silva ${ }^{2}$, N.S. Dias ${ }^{3,4}$ and J.H.Correia ${ }^{1}$ \\ ${ }^{1}$ Department of Industrial Electronics, University of Minho, Campus of Azurem, 4800-058 Guimaraes, Portugal \\ ${ }^{2}$ MIT Portugal Program, University of Minho, School of Engineering, Campus of Azurem, Guimarães, Portugal \\ ${ }^{3}$ Life and Health Sciences Research Institute (ICVS), University of Minho, Campus of Gualtar, Braga, Portugal \\ ${ }^{4}$ DIGARC, Portugal Polytechnic Institute of Cavado and Ave, Barcelos, Portugal
}

\begin{abstract}
A neural electrode array design is proposed with $3 \mathrm{~mm}$ long sharpened pillars made from an aluminumbased substrate. The array is composed by 25 electrically insulated pillars in a 5 x 5 matrix, in which each aluminum pillar was precisely machined via dicing saw technique. The result is an aluminum structure with high-aspect-ratio pillars (19:1), each with a tip radius of $10 \mu \mathrm{m}$. A thin-film of platinum was deposited via sputtering technique to perform the ionic signal transduction. Each pillar was encapsulated with an epoxy resin insulating the entire pillar excluding the tip. This process resulted in mechanically robust electrodes each capable of withstanding loads up to $200 \mathrm{mN}$ before bending. The array implantation tests were conducted on agar gel at speeds of $50 \mathrm{~mm} / \mathrm{s}, 120 \mathrm{~mm} / \mathrm{s}$ and $180 \mathrm{~mm} / \mathrm{s}$ which resulted in average implantation forces of $119 \mathrm{mN}, 145 \mathrm{mN}$ and $150 \mathrm{mN}$ respectively. Insertion and withdrawal tests were also performed in porcine cadaver brain showing a necessary force of $66 \mathrm{mN}$ for successful explantation. A three point flexural test demonstrated a displacement of $0.8 \mathrm{~mm}$ before array's breakage. The electrode's impedance was characterized showing a near resistive impedance of $385 \Omega$ in the frequency range from $2 \mathrm{kHz}$ to $125 \mathrm{kHz}$. The resultant array, as well as the fabrication technique, is an innovative alternative to silicon-based electrode solutions, avoiding some fabrication methods and limitations related to silicon and increasing the mechanical flexibility of the array.
\end{abstract}

Keywords: Aluminum; neural electrode; microfabrication; dicing; flexible.

\section{Introduction}

The ability to access areas of the brain in order to record neural electrical activity or to perform functional stimulation is a key feature to understand the neurophysiological processes and to restore the nervous system's lost functionalities [1]. Advances in microtechnologies allowed the development of micrometer size instruments to interact with neural tissue. These instruments, known as neural electrodes, are usually invasive, of small size, and with multiple channels.

Currently, there are many neural electrode approaches and designs [2]. The Michigan probe [3] and the Utah array [4] stand out due to their high-density of electrodes, tridimensional nature and efficacy in long term recording.

These two approaches are based on silicon microfabrication. This implies that their fabrication is heavily dependent on CMOS technology, which is readily and easily available due to the massification of consumer electronics. Due to this fact, there's a significant advantage on using standard microfabrication techniques. However, this implies that the entire process will be based on silicon.

Despite the many advantages of silicon, brittleness stands out as a major disadvantage when interacting with biological tissues. A logical evolution towards bio-integration would be the use of materials with superior flexibility than silicon.

Both arrays are commercially available but are limited to cortical regions, due to the size of the electrodes being restricted to $1.5 \mathrm{~mm}$. Longer electrodes have been achieved by using $\mu$-wire electrical discharge machining [5]. Longer electrodes like these would allow the exploration of other brain structures of great importance for neurophysiology, such as the hippocampus [6]. Rats are commonly used as animal

*Corresponding author: Tel:+351967947793

E-mail address: ID3251@ alunos.uminho.pt (A.C.Peixoto) 
models by neurobiologists to better understand the human brain [7]. In rats the hippocampus is situated between 2 to $4 \mathrm{~mm}$ below the surface [8]. In this paper we describe an array consisting of 25 electrodes, each able to reach $3 \mathrm{~mm}$ below the brain surface. At this depth the electrodes are able to reach the hippocampus. Aluminum was used as structural material and also as electrical conductor for the acquired signals. Epoxy resin was used as encapsulation and also as structural adhesive between micropillars. The combination of these two materials allows greater flexibility than silicon. A sputtered thin-film of platinum was used as the transduction layer between signal acquisition electronics and neural activity. By introducing materials with higher degree of flexibility than silicon, this approach decreased the mechanical mismatch between the brain and the array, reducing possible trauma due to brain dynamics [9]. By avoiding some advanced microfabrication techniques such as reactive ion etching and chemical vapor deposition, the present approach is more cost-effective than the two aforementioned alternatives. Although using alternative materials, the processes are based on standard microfabrication techniques, this means that the arrays can be manufactured in batches with a throughput yield similar to silicon. The fabrication steps are described in detail, the array is morphologically and mechanically characterized, and the platinum thin-film electrodes are electrochemically characterized.

This paper also offers a significant improvement in several aspects over a previously described aluminum array [10]. The present encapsulation process relies on the deposition and selective removal of the resin around each pillar, while in the previous version an anodizing process was used. While the present approach performs a uniform and sealed layer the previous relies on aluminum oxide, which has nanopores that are inherent of the anodizing process [11]. Also, the present approach is simpler since it avoids the need of a special set-up to inject a controlled current from the array to the cathode while submerged in and acidic solution [12]. The reproducibility is increased in the present version, because it relies on the precision of the dicing machining to deinsulate the tips and control the electrode's active area. The new array has a density of 2.8 electrodes per $\mathrm{mm}^{2}$ which is more than double the density of the previous version. Higher electrodes densities allows higher spatial resolution of the brain activity, which is of great value to better understand brain functions [13]. Finally, the pillars' aspect-ratio almost doubled going from 12:1 to 19:1. This reduction in the pillar's cross-section is very important since it is crucial for neural electrodes to displace the minimum quantity of neural tissue [6].

\section{Array Design}

Due to the various applications of invasive electrodes in neurophysiology, the array's design should be made according to a specific application. Arrays with high-density are preferred when used for applications such as neuroprosthetics or for mapping the interaction between diverse brain's regions $[2,10]$. The described array has 25 electrodes, one at each tip of a micropillar, with a spacing of $600 \mu \mathrm{m}$ between each, resulting in an electrode density of 2.8 electrodes per $\mathrm{mm}^{2}$.

It is also important that the micropillars cause minimum tissue trauma due to insertion and subsequent tissue displacement. It was a major concern to design a probe that would displace the smallest volume of brain tissue during insertion. This implies that the pillars should have the smallest cross section possible. Each micropillar has a cross section of $160 \mu \mathrm{m} \times 160 \mu \mathrm{m}$ and $3 \mathrm{~mm}$ of length, which results in a high-aspectratio of 19:1. Each micropillar has a pyramidal profile with a tip radius of $10 \mu \mathrm{m}$. These sharp tips reduce the insertion force for penetration, subsequently reducing insertion trauma by minimizing tissue dimpling and compression [15].

The thin and long profile of the pillars requires that their mechanical properties should withstand the axial forces needed for penetration without buckling or breaking, as well as to withstand the shear stresses due to imperfect insertion, namely, angled penetration and movement during penetration. Each pillar consists of a combination of an aluminum pillar and a thick epoxy coating all around the pillar. The aluminum has a compressive strength of approximately $70 \mathrm{~N} / \mathrm{mm}^{2}$ while the epoxy has a compressive strength of $65 \mathrm{~N} / \mathrm{mm}^{2}$ $[12,13]$. Although with a significantly smaller compressive and shear strength than silicon, aluminum and epoxy offer superior strength than the soft and viscoelastic brain tissue (Table 1). Also the penetration forces of cylindrical probes ranging from $100 \mu \mathrm{m}$ to $200 \mu \mathrm{m}$ in diameter have been reported to be below $15 \mathrm{mN}$ in 
the rat brain [15]. For this magnitude of forces, both materials have the mechanical properties to withstand penetration without bending, as shown in the results section. In the case of imperfect insertions, the probes can be subjected to shear forces, which could bend them and lead to breakage. Aluminum and epoxy are ductile materials; this property gives the probes a greater degree of flexion than silicon.

Signal quality is of great importance, since neural signal have a magnitude of power in the order of few micro-watts. At this scale, signal attenuation due to electrical resistance becomes important, therefore the conducting material should have the least possible amount of resistance. Aluminum is inherently an excellent electrical conductor with resistivity values of approximately $26.9 \mathrm{n} \Omega . \mathrm{m}$, which makes it an appropriate choice for conducting electrical signals [17]. This characteristic offers an electrical advantage over doped silicon, which has been reported to have a resistivity of $50 \mu \Omega . \mathrm{m}$ [18], which is a difference of 3 orders of magnitude between aluminum and doped silicon. Table 1 compares the different materials properties.

Table 1 - Material properties comparison

\begin{tabular}{llllll}
\hline Parameter & Unit & Brain[19] & Aluminum[17] & $\begin{array}{l}\text { Hysol } \\
\mathbf{3 4 3 0 [ 1 6 ]}\end{array}$ & Silicon[20] \\
\hline Young's Modulus & $\mathrm{GPa}$ & $19 \times 10^{-6}$ & 70 & 3.21 & 190 \\
Shear strength & $\mathrm{MPa}$ & --- & 75 & --- & 7000 \\
$\begin{array}{l}\text { Resistivity } \\
\begin{array}{l}\text { Compressive } \\
\text { strength }\end{array}\end{array}$ & $\mathbf{S} . \mathrm{m}$ & --- & $2.69 \times 10^{-8}$ & $3 \times 10^{15}$ & $* 700 \times 10^{-6}$ \\
$\begin{array}{l}\text { Cost } \\
\text { S }\end{array}$ & $16 \times 10^{-3}$ & 70 & 65 & 8300 \\
\hline
\end{tabular}

*Highly doped silicon [18].

It was necessary to select a material to perform the ionic transduction at each tip since aluminum is not adequate to perform the charge transfer at the electrode-electrolyte interface. Platinum has been widely used as a neural electrode for recording and stimulation, with evidences of chemical stability in saline, highly polarizable, and with relative high charge-injection limits $[18,19,20]$. A thin-film of Platinum was deposited via DC sputtering at each electrode, allowing the transduction of electrical signals from the neuronal tissue. Figure 1 shows a perspective view of the final array.

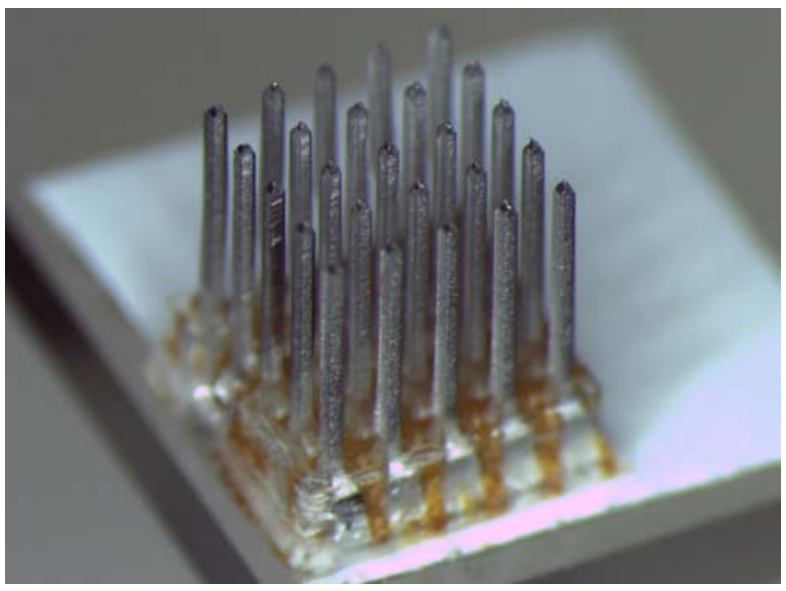

Figure 1 - Perspective view of the 5 x 5 electrode neural array. 


\section{Array Fabrication}

The fabrication process for the proposed electrode design used a combination of standard CMOS processes such as wafer dicing, wet-etching, thin-film deposition; and also non-standard processes such as microcasting and adhesive bonding. Although the wafer dicing is usually performed on silicon, our process technology applied it for dicing aluminum and epoxy resin.

All dicing steps were performed on a Disco DAD 2H/6T dicing machine, equipped with Disco ZHDG blades capable of performing $3 \mathrm{~mm}$ deep, $350 \mu \mathrm{m}$ wide cuts and $\mathrm{V}$-shaped grooves. The substrate used was a $99 \%$ pure aluminum block, $25 \mathrm{~mm}$ wide and $4 \mathrm{~mm}$ thick (Figure 2a). The first series of cuts were performed on one of the surfaces that we will define as the "backside" of the array. These cuts outlined the pads' region. These pads made the electrical connection between each electrode tip and the acquisition electronics. The cuts have a grid pattern with a spacing of $600 \mu \mathrm{m}$ and depth of $1 \mathrm{~mm}$ (Figure 2b). Afterwards, the resultant cavities were filled with an epoxy resin (Hysol 9492) that serves as electrical insulation between each pillar, individualizing all the electrodes and also acting as the structural bond between pillars [24]. The polymer was injected over the backside, forcing out any air remaining inside the grooves and replacing the empty space between pads with the epoxy resin. After the curing of the polymer, the backside surface was abraded with small grit sandpaper to remove the excess polymer, resulting in a smooth surface (Figure 2c).

The epoxy resin was selected as the bonding material due to its high-level of adhesion to aluminum, excellent electrical insulation and chemical resistance [25].

With the backside defined, the substrate was turned over in order to create the pillars. A program was performed to cut a matrix of $3 \mathrm{~mm}$ deep and $250 \mu \mathrm{m}$ wide pillars (Figure 2d). Each pillar was centered with each pad. After this dicing stage, a significant amount of burr was formed at the pillars' edges. In order to remove the burr and also to decrease the pillars' cross section, the array was immersed in $20 \mathrm{~mL}$ of aluminum etchant at $50{ }^{\circ} \mathrm{C}$ for 70 minutes [26]. The etchant was constantly circulating through the pillars with the aid of a magnetic stirrer. As a result of the etching step, extremely thin aluminum pillars were achieved (Figure 2e). The pillars' dimensions will be discussed in further detail in the next section. Although visually, the epoxy appeared to be slightly discolored by the etching bath, it remained strong enough for normal handling.

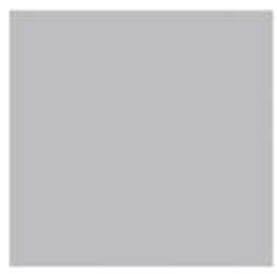

a)

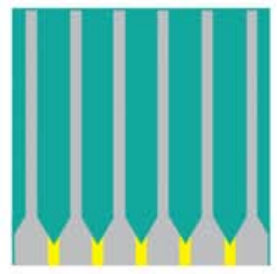

f)

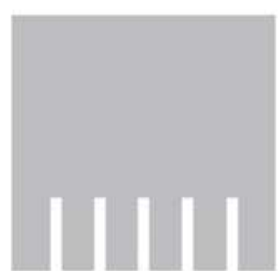

b)

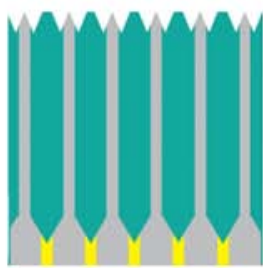

g)

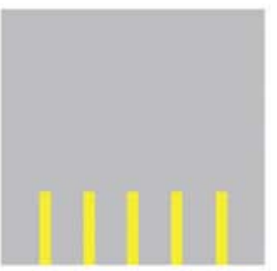

c)

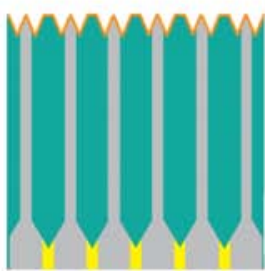

h)

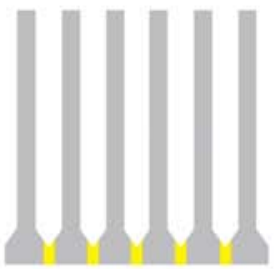

d)

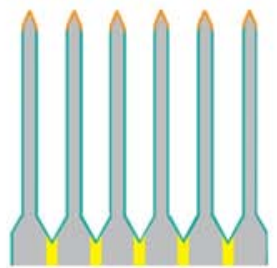

i)

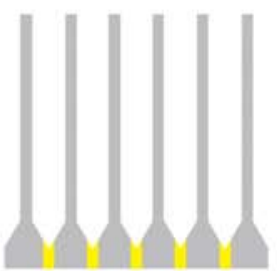

e)

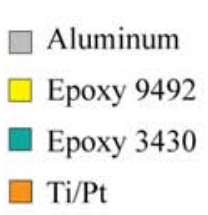

$\mathrm{Ti} / \mathrm{Pt}$

Figure 2 - Fabrication Steps. 
A second step of casting was performed, this time to completely fill the space between pillars with epoxy resin (Figure 2f). The next cutting step had a twofold purpose: one was to sharpen the pillars' tips to facilitate insertion into the brain tissue and the second was to guarantee the deposition of the Platinum thinfilm only on the tips. The tips were formed by passing a V-shaped blade on the top of each pillar (Figure 2g).

In the resulting surface was deposited a layer of $70 \mathrm{~nm}$ of Titanium (Ti) followed by a $200 \mathrm{~nm}$ layer of Platinum (Pt). The Pt layer will allow an efficient charge transfer between the electrode and electrolyte (neural tissue) while $\mathrm{Ti}$ serves as an adhesive layer. The $\mathrm{Ti}$ layer was deposited using electron beam evaporation at a starting pressure of $2.8 \times 10^{-6} \mathrm{mBar}$ while supplying a current of $60 \mathrm{~mA}$ and a potential of $7 \mathrm{kV}$. The duration of the deposition was 5 minutes and the maximum temperature measured at the substrate was $60^{\circ} \mathrm{C}$. The Pt layer deposited using DC sputtering at a starting pressure of $3.3 \times 10^{-6} \mathrm{mBar}$ while supplying a current of $60 \mathrm{~mA}$ and a potential of $7 \mathrm{kV}$. The duration of the deposition was 3 minutes and the maximum temperature measured at the substrate was $45^{\circ} \mathrm{C}$ (Figure $2 \mathrm{~h}$ ).

Afterwards, a third step of dicing was performed to remove the excess epoxy, resulting in the highaspect-ratio pillars. The resulting pillars have a core of aluminum to serve as a pathway for neural signals to be transmitted from the tips to the pads. The epoxy layer serves as an electrical insulator, blocking the aluminum core to receive unwanted neural activity (Figure 2i). Photos of the fabrication steps are shown in Figure 3.
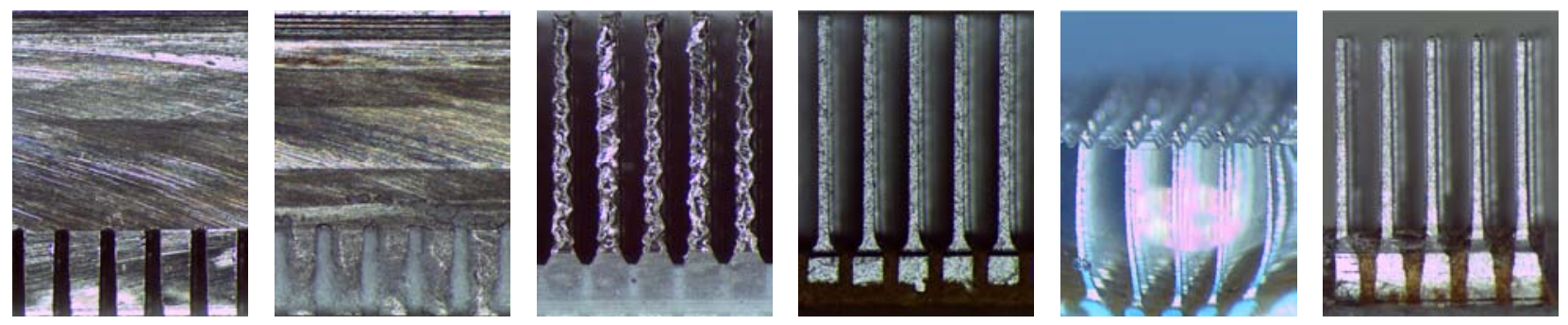

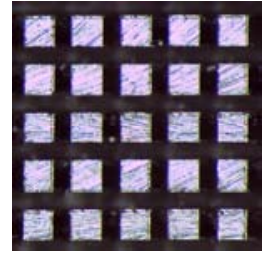

a)

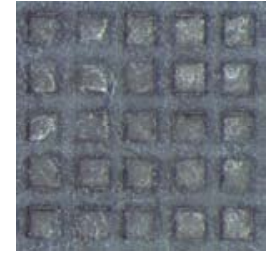

b)

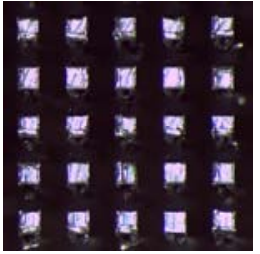

c)

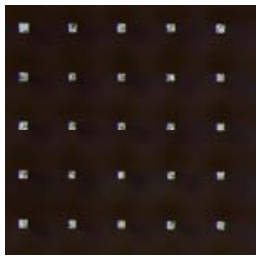

d)

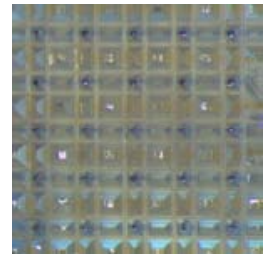

e)

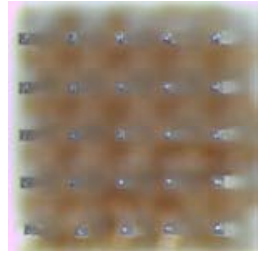

f)

Figure 3 - Structure fabrication. First row photos correspond to the side view and second row photos correspond to either top or bottom view: a) Aluminum substrate with pads region delimitation; b) Pads' grooves filled with epoxy resin; c) Pillars after dicing stage; d) Pillars after the wet-etching step; e) Casted array with tips (optical distortion due to the convex shape of the epoxy); f) Final array structure.

\section{Results}

\subsection{Morphological}

A total of 4 arrays were used for the measurement of the array's dimensions. After the wet etching process the aluminum pillars were measured to verify the variation that the corrosion introduces to their main dimensions. In a population of 20 samples, the average length was $3.09 \mathrm{~mm}$ with a standard deviation of $0.06 \mathrm{~mm}$. Since aluminum is ductile and each cut creates small amounts of burr at the pillars' edges, it was important to observe the different widths between the first and second series of cuts when creating the 
pillars (Figure 3c). These cuts are perpendicular so there should be a small difference in widths between two perpendicular sides of each pillar. For these measurements 20 samples positioned at the perimeter of each array were used. All widths were measured at middle height of each pillar. The average width was $117 \mu \mathrm{m}$ for the first direction and $129 \mu \mathrm{m}$ for the second direction; both standard deviations were of $7 \mu \mathrm{m}$. The width difference between the two directions showed no statistical significance ( $\mathrm{p}<0.05$; T-test). Figure 4 shows the box plot for each group of measurements.

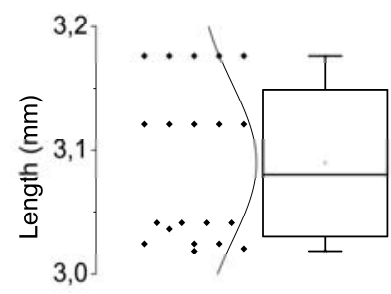

a)
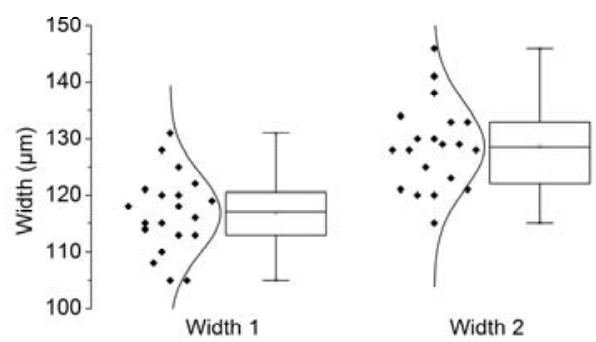

b)

Figure 4- Box plot with normal distribution of the aluminum pillars after wet-etching: a) Length of the pillars; b) Width of the pillars in first direction and second direction (width 1 and width 2).

Figure 5 shows the main dimensions that define each pillar with the epoxy coating. The pillars are $3 \mathrm{~mm}$ long and $160 \mu \mathrm{m}$ wide, with a pyramidal shaped tip. The encapsulating epoxy is approximately $20 \mu \mathrm{m}$ thick. We have achieved alignment accuracies of $30 \mu \mathrm{m}$ between the wafer's upper and back sides and of $6 \mu \mathrm{m}$ between the epoxy layer and the aluminum pillar. The level of accuracy depends on the optical magnification of the dicer's microscope.

The measured tip radius was $10 \mu \mathrm{m}$. The pillars' base is an aluminum block of $400 \mu \mathrm{m} \times 400 \mu \mathrm{m} \times 750 \mu \mathrm{m}$. The tip measurement was performed in a FEI Nova NanoSem ${ }^{\mathrm{TM}} 200$. All other measurements were performed with Leica $\mathrm{M} 80^{\mathrm{TM}}$ stereo microscope and Leica LAS ${ }^{\mathrm{TM}}$ software.

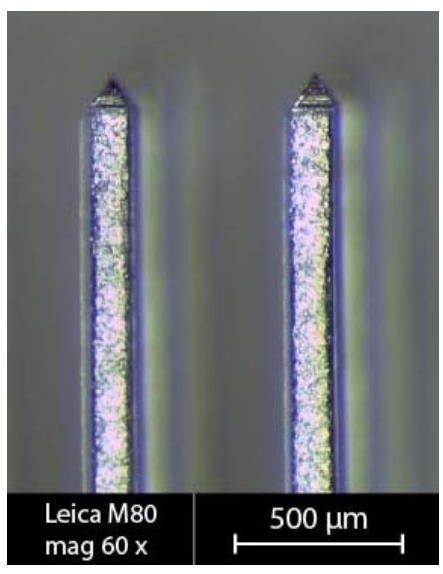

a)

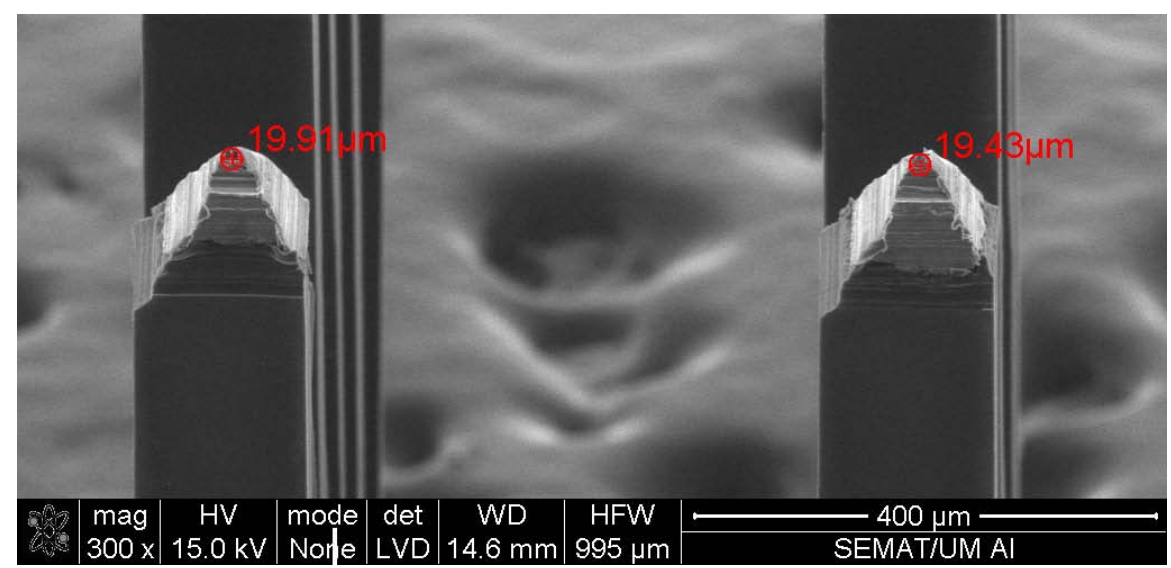

b)

Figure 5 - Detailed view of the final pillars: a) Pillars lateral view coated with an epoxy layer, b) Tip morphology. 


\subsection{Mechanical}

The mechanical tests focused on the force required to implant and explant the array on agar brain phantom and also on porcine cadaver brain. The tests were performed on a Shimadzu AG-IS dynamometer equipped with a $50 \mathrm{~N}$ load cell capable of a $5 \mathrm{mN}$ resolution. Agar gel has been widely used as an in vitro alternative for brain tissue [27,28,29]. The insertion mechanics were tested in $0.5 \%$ agar gel which mimics the brain cortex. Figure 6a) shows the setup arrangement for the implantation tests. The array was attached to the dynamometer shaft, which moved downward towards the agar gel at a predetermined speed. The shaft was set to move at three different speeds, namely, $180 \mathrm{~mm} / \mathrm{s}, 120 \mathrm{~mm} / \mathrm{s}$ and $50 \mathrm{~mm} / \mathrm{s}$. Figure $6 \mathrm{~b}$ ) shows the force applied versus the array's displacement towards the gel.

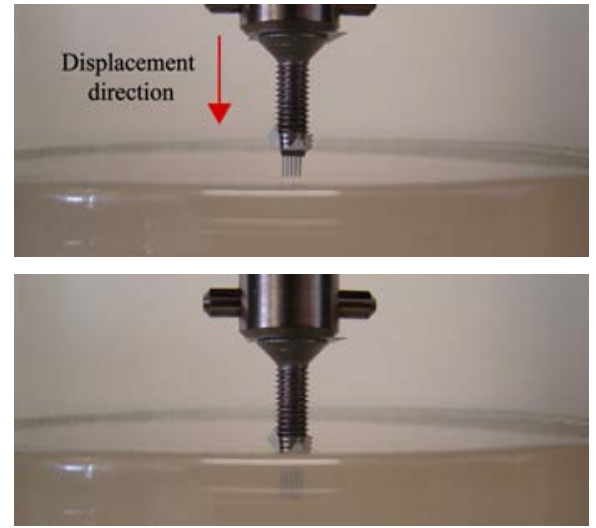

a)

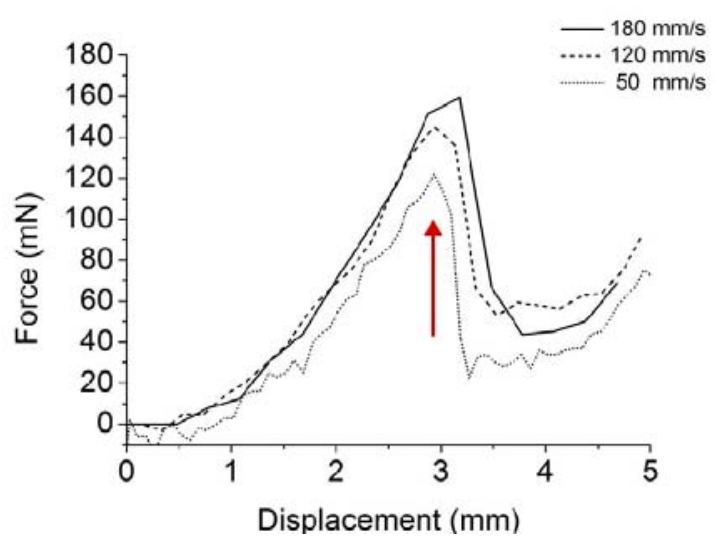

b)

Figure 6 - Mechanical insertion tests on $0.5 \%$ agar gel: a) Insertion setup. Top picture, array before implantation, Bottom picture, array fully implanted; b) Force versus displacement for the three tested speeds. The penetration moment is highlighted with a red arrow.

In Figure 6b) was observed an increase on the force applied on the initial stage while still subjected to the gel's resistance until the moment where the electrodes' tips appear to pierce the surface. Beyond this moment, as expected, there was an abrupt drop in the required load. After the electrode's full penetration there was a slight increase in the curve's slope, indicating that the array's base was pushing against the gel. It could be concluded that the maximum force coincided with the necessary load for piercing the gel and beginning implantation. By increasing the implantation speed, the force needed to penetrate the agar increased as well. The acquired data is summarized on Table 2.

Table 2 - Statistical data of the implantation tests.

\begin{tabular}{lllll}
\hline $\begin{array}{l}\text { Implantation } \\
\text { Speed }(\mathbf{m m} / \mathbf{s})\end{array}$ & $\begin{array}{l}\text { Number of } \\
\text { samples }\end{array}$ & $\begin{array}{l}\text { Average } \\
\text { Maximum } \\
\text { Force }(\mathbf{m N})\end{array}$ & $\begin{array}{l}\text { Standard } \\
\text { Deviation }(\mathbf{m N})\end{array}$ & $\begin{array}{l}\text { Average } \\
\text { Maximum Force } \\
\text { per electrode } \\
(\mathbf{m N})\end{array}$ \\
\hline 50 & 4 & 119 & 8 & 4.76 \\
120 & 4 & 145 & 8 & 5.8 \\
180 & 4 & 150 & 2 & 6 \\
\hline
\end{tabular}


Between the three tested speeds there is a maximum variation of force of $1.24 \mathrm{mN}$ per electrode. The average implantation force is considerably lower than our previous thicker probe [10] and in the same order of magnitude of Das et al. [29]. Implantation tests with similar parameters as the agar gel were performed on porcine cadaver brain with the purpose of testing the array robustness, these tests showed significant tissue dimpling without implantation success. Penetration of the dura mater and subsequent implantation on the brain was only possible when displacements of four times the length of the electrodes were applied, which suggests that for a high-density electrode matrix it is necessary the use of impact insertion techniques to avoid massive tissue dimpling and compression before penetration [30]. Successful implantation could only be verified through explantation data, since it could not be observed from the penetration curve. Figure 7a) shows the withdrawal data on porcine cadaver brain while Figure $7 \mathrm{~b}$ ) shows the data related to the agar gel. Each figure shows the key moment highlighted by a red arrow. Moment 1 is where the brains' surface is in its relaxed state, in moment 2 there is a maximum tension applied and in moment 3 there is a complete detachment between the array and the tested material. Moment 1 happens because the array goes from a state of actively compressing the brain tissue to a relaxation state. The rise in tension during withdrawal is due to the drag forces between the electrodes and the surrounding material. This could be observed clearly in the cadaver brain as it followed the array trajectory originating a temporary protuberance in the region of implantation (moment 2). This was followed by a drop in the applied tension which led to the instant where the array was completely detached from the brain (moment 3 ). For the brain, the difference in force between brain relaxation and maximum tension was of $66 \mathrm{mN}$. This suggests that the array was fully implanted prior to removal. Besides this analysis the full implantation in agar could also be observed visually since it was Figure 6a) bottom.

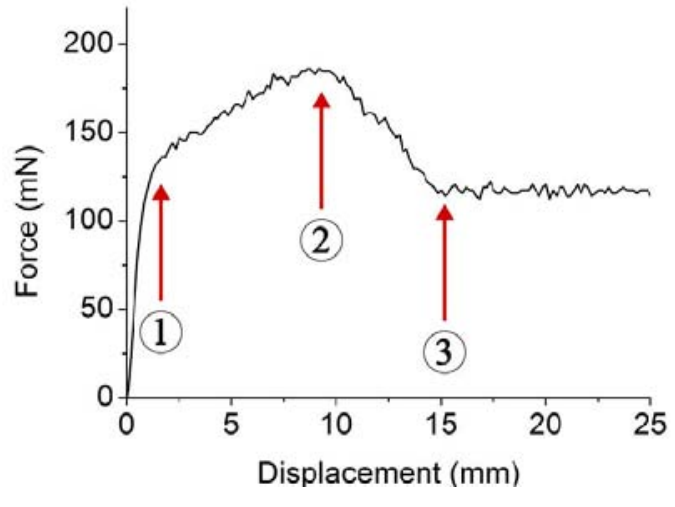

a)

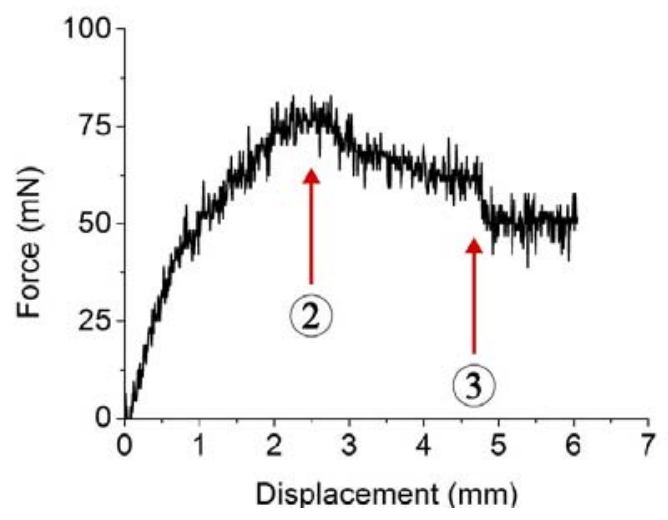

b)

Figure 7 - Withdrawal results with key moments highlighted by red arrows. Moment 1, brain relaxation; Moment 2, maximum tension; Moment 3 complete detachment; a) Porcine cadaver brain; b) Agar gel.

A destructive test was performed against a glass substrate to acquire the force needed to bend the pillars. At a displacement rate of $82 \mu \mathrm{m} / \mathrm{s}$, the measured buckling load was $5 \mathrm{~N}$. This buckling load is inferior to silicon probes since aluminum has inferior Young's modulus [31].

A flexibility test was performed using the three point flexural test. The tested sample was $20 \mathrm{~mm}$ wide and $9.6 \mathrm{~mm}$ long. The support was made of aluminum with $15 \mathrm{~mm}$ spacing between support points (Figure 8a). A downward force was applied in the middle of the sample with a cylindrical aluminum support until sample rupture could be observed. A maximum deflection before rupture of $0.8 \mathrm{~mm}$ was achieved (Figure $8 b)$. 


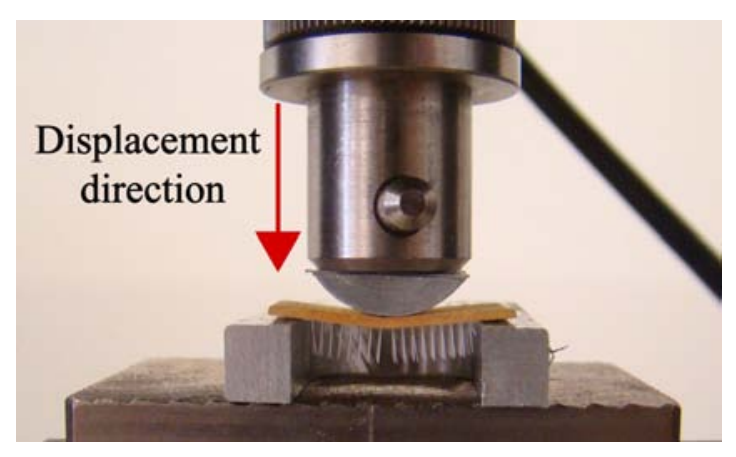

a)

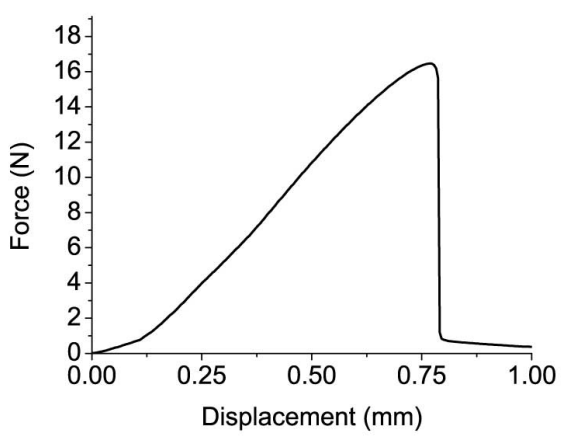

b)

Figure 8 - Three point flexural test set up and results; a) Aluminum base with $15 \mathrm{~mm}$ spacing between support points. The picture was taken at the moment of rupture. b) Force versus displacement graph.

\subsection{Electrochemical}

The electrochemical impedance characteristics of the Platinum thin-film were obtained using a Gamry Reference $600^{\mathrm{TM}}$ Potentiostat/Galvanostat. A three-electrode chemical cell configuration with an $\mathrm{Ag} / \mathrm{AgCl}$ reference electrode and a large area Platinum counter electrode were used. The corresponding area of the Platinum thin-film tested as the working electrode was of $38.4 \mathrm{~mm}^{2}$. The electrolyte used was a saline solution with a concentration of $0.9 \%$ of $\mathrm{NaCl}$. The experiment was carried out at room temperature. The alternate current excitation voltage was $10 \mathrm{mV}$ with a frequency range from $1 \mathrm{~Hz}$ to $1 \mathrm{MHz}$. Figure 9a) shows the Bode plot of the impedance magnitude versus frequency for the Platinum thin-film. Three readings of the same sample were performed for increased measurement reliability. At $1 \mathrm{kHz}$ the average impedance was $385 \Omega$. The impedance at this specific frequency is of neurobiological interest because the neuronal cell's action potential has a duration close to $1 \mathrm{~ms}$, and therefore, provides the attenuation introduced by the electrode for this range of frequencies. The electrode exhibits a near-resistive phase angle in the frequency range between $2 \mathrm{kHz}$ to $125 \mathrm{kHz}$ (Figure 9b), and a capacitive behavior for the remaining frequencies.

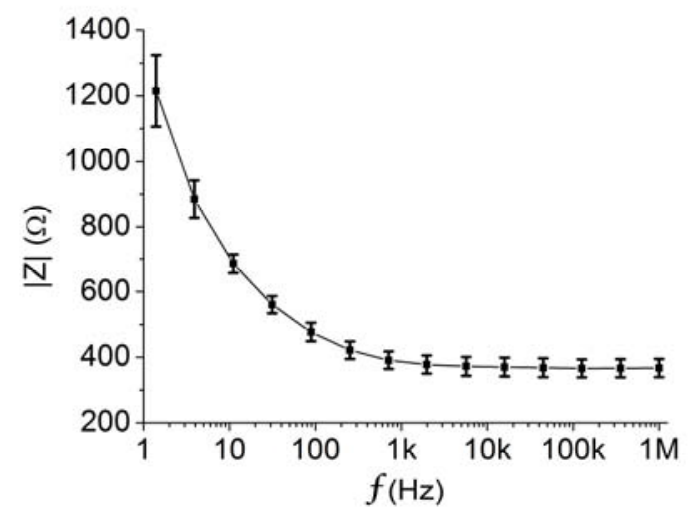

a)

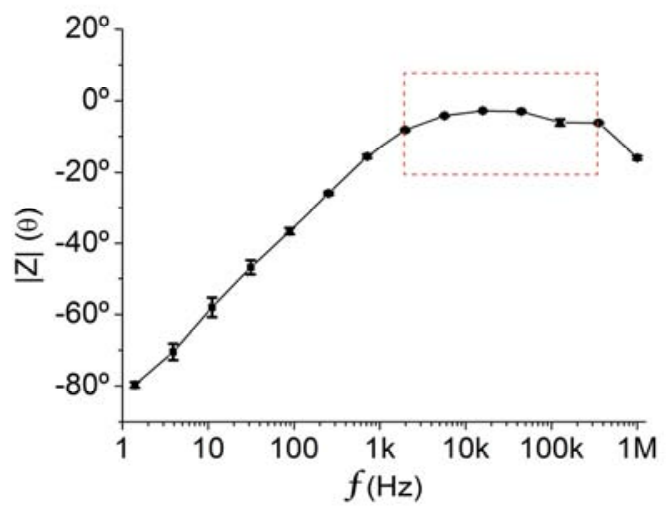

b)

Figure 9 - Bode plot of the electrochemical impedance spectroscopy of the electrode in saline solution: a) Magnitude; b) Phase angle with highlight of the near resistive region. 


\section{Conclusions}

The present work demonstrated that despite the challenges of micromachining aluminum, it is possible to fabricate high-aspect-ratio structures employing only dicing and wet-etching techniques. The aluminumbased substrate proved to be a possible alternative to silicon-based arrays by offering some advantages in terms of flexibility and electrical conductivity. The fabrication approach is cost-effective by avoiding advanced microfabrication techniques. Although the wet-etching step introduces some variations in the pillar's width, the encapsulating process corrects these variations by relying in the dicing machine's high resolution. The electrode array has the ability to penetrate deeper than commercially available arrays and is not subject to breakage during insertion. The result was a robust invasive neural electrode array with $3 \mathrm{~mm}$ long pillars with the ability to penetrate agar brain phantom and porcine cadaver brain tissue. Results showed that increasing implantation speeds would increase penetration forces in agar gel proportionally. The three point flexural test showed the ability of the array to bend made possible by the combination of aluminum and epoxy resin at its base. The platinum coating demonstrated through the electrochemical tests in saline solution to have the required performance for neural tissue interface. The present fabrication method can be applied to other metals, allowing alternate structural materials instead of silicon in the design of neural array. Overall, the proposed design and fabrication procedure is an eligible alternative for neural signal recording and stimulation systems and also offers a contribution to the future of flexible implantable devices.

\section{Acknowledgment}

A. C. Peixoto is supported by the Portuguese Foundation for Science and Technology under grant SFRH/BD/89509/2012. This work was supported by FCT with the reference project FCOMP $010124-$ FEDER-010909 (FCT/PTDC/SAU-BEB/100392/2008). The authors wish to thank Professor Senentxu Lanceros-Mendez for kindly granting access to the Shimadzu dynamometer and also to Pedro Libânio Martins for the valuable suggestions and technical assistance during the implantation tests.

\section{References}

[1] R. A. Normann, "Technology insight: future neuroprosthetic therapies for disorders of the nervous system," Nat. Clin. Pract. Neurol., vol. 3, no. 8, pp. 444-452, 2007.

[2] K. C. Cheung, "Implantable microscale neural interfaces," Biomed. Microdevices, vol. 9, no. 6, pp. 923-38, Dec. 2007.

[3] K. D. Wise, "Wireless integrated microsystems: Wearable and implantable devices for improved health care," in Solid-State Sensors, Actuators and Microsystems Conference, 2009, pp. 1-8.

[4] A. Sharma, L. Rieth, P. Tathireddy, R. Harrison, H. Oppermann, M. Klein, M. Töpper, E. Jung, R. A. Normann, G. Clark, and F. Solzbacher, "Long term in vitro functional stability and recording longevity of fully integrated wireless neural interfaces based on the Utah Slant Electrode Array," $J$. Neural Eng., vol. 8, no. 4, Aug. 2011.

[5] P. Tathireddy, D. Rakwal, E. Bamberg, and F. Solzbacher, "Fabrication of 3-dimensional silicon microelectrode arrays using micro electro discharge machining for neural applications," in Solid-State Sensors, Actuators and Microsystems Conference. Transducers, 2009, pp. 1206-1209.

[6] J. Csicsvari, D. A. Henze, B. Jamieson, K. D. Harris, A. Sirota, P. Barthó, K. D. Wise, and G. Buzsáki, "Massively parallel recording of unit and local field potentials with silicon-based electrodes," J. Neurophysiol., vol. 90, no. 2, pp. 1314-1323, 2003. 
[7] N. A. Fineberg, M. N. Potenza, S. R. Chamberlain, H. A. Berlin, L. Menzies, A. Bechara, B. J. Sahakian, T. W. Robbins, E. T. Bullmore, and E. Hollander, "Probing compulsive and impulsive behaviors, from animal models to endophenotypes: a narrative review," Neuropsychopharmacology, vol. 35, no. 3, pp. 591-604, Feb. 2010.

[8] G. Paxinos and C. Watson, The Rat Brain in Stereotaxic Coordinates - Part 1, Sixth Edit. Elsevier Inc., 2007.

[9] T. Kim, J. G. McCall, Y. H. Jung, X. Huang, E. R. Siuda, Y. Li, J. Song, Y. M. Song, H. a. Pao, R.-H. Kim, C. Lu, S. D. Lee, I.-S. Song, G. Shin, R. Al-Hasani, S. Kim, M. P. Tan, Y. Huang, F. G. Omenetto, J. a. Rogers, and M. R. Bruchas, "Injectable, Cellular-Scale Optoelectronics with Applications for Wireless Optogenetics," Science, vol. 340, no. 6129, pp. 211-216, Apr. 2013.

[10] A. C. Peixoto, S. B. Goncalves, A. F. Silva, N. S. Dias, and J. H. Correia, "Neural Electrode Array Based on Aluminum: Fabrication and Characterization," IEEE Sens. J., vol. 13, no. 9, pp. 3319-3324, 2013.

[11] J. Zahavi and M. Metzger, "Electron Microscope Study of Breakdown and Repair of Anodic Films on Aluminum,” J. Electrochem. Soc., vol. 119, no. 11, p. 1479, 1972.

[12] J. Yahalom and T. P. Hoar, "Galvanostatic anodizing of aluminium," Electrochim. Acta, vol. 15, no. 6, pp. 877-884, Jun. 1970.

[13] M. A. Nicolelis, a a Ghazanfar, B. M. Faggin, S. Votaw, and L. M. Oliveira, "Reconstructing the engram: simultaneous, multisite, many single neuron recordings.," Neuron, vol. 18, no. 4, pp. 529-37, Apr. 1997.

[14] G. Buzsáki, "Large-scale recording of neuronal ensembles.," Nat. Neurosci., vol. 7, no. 5, pp. 446-51, May 2004.

[15] A. A. Sharp, A. M. Ortega, D. Restrepo, D. Curran-Everett, and K. Gall, "In vivo penetration mechanics and mechanical properties of mouse brain tissue at micrometer scales," IEEE Trans. Biomed. Eng., vol. 56, no. 1, pp. 45-53, Jan. 2009.

[16] Henkel AG \& Co., "Hysol® 3430 ${ }^{\mathrm{TM}}$ Technical Datasheet," 2008. [Online]. Available: http://www.loctite.co.uk/fullproduct-list-loctite-4995.htm? countryCode=uke\&BU=industrial \&parentredDotUID=productfinder\&redDotUID=1000000IC7Z.

[17] R. Cobden and A. Banbury, "Aluminium: Physical Properties, Characteristics and Alloys," TALAL. p. 60, 1994.

[18] R. Sharma, P. Tathireddy, S. Lee, and L. Rieth, "Application-specific customizable architectures of Utah neural interfaces," Procedia Eng., vol. 25, pp. 1016-1019, 2011.

[19] B. Rashid, M. Destrade, and M. Gilchrist, "Mechanical characterization of brain tissue in compression at dynamic strain rates," J. Mech. Behav. Biomed. Mater., 2012.

[20] K. E. Petersen, "Silicon as a mechanical material," Proc. IEEE, vol. 70, no. 5, pp. 420-457, 1982. 
[21] S. F. Cogan, "Neural stimulation and recording electrodes," Annu. Rev. Biomed. Eng., vol. 10, pp. 275-309, Jan. 2008.

[22] S. B. Brummer, L. F. Robblee, and F. T. Hambrecht, "Criteria for selecting electrodes for electrical stimulation: theoretical and practical considerations," Ann. N. Y. Acad. Sci., vol. 405, pp. 159-171, 1983.

[23] M. R. Neuman, "Biopotential Electrodes," in The Biomedical Engineering Handbook: Second Edition, 2000.

[24] Henkel AG \& Co., "Hysol® 9492 ${ }^{\mathrm{TM}}$ Technical Datasheet," 2007. [Online]. Available: http://www.loctite.co.uk/fullproduct-list-loctite-4995.htm?countryCode=uke \&BU=industrial \&parentredDotUID=productfinder\&redDotUID=1000000J9BL.

[25] DSM Engineering Plastics, "Gluing Guide," 2013. [Online]. Available: www.dsm.com/content/dam/dsm/automotive/en_US/documents/gluing-guide.pdf.

[26] Transene.Co.Inc., "Material safety data sheet for aluminum etchant Type A," Rowley, MA, 1987.

[27] Z.-J. Chen, G. T. Gillies, W. C. Broaddus, S. S. Prabhu, H. Fillmore, R. M. Mitchell, F. D. Corwin, and P. P. Fatouros, "A realistic brain tissue phantom for intraparenchymal infusion studies," $J$. Neurosurg., vol. 101, no. 2, pp. 314-322, 2004.

[28] R. Pomfret, G. Miranpuri, and K. Sillay, "The Substitute Brain and the Potential of the Gel Model," Ann. Neurosci., vol. 20, no. 3, Jul. 2013.

[29] R. Das, D. Gandhi, S. Krishnan, L. Saggere, and P. J. Rousche, "A benchtop system to assess cortical neural interface micromechanics.," IEEE Trans. Biomed. Eng., vol. 54, no. 6 Pt 1, pp. 1089-96, Jun. 2007.

[30] P. J. Rousche and R. a Normann, "A method for pneumatically inserting an array of penetrating electrodes into cortical tissue.," Ann. Biomed. Eng., vol. 20, no. 4, pp. 413-22, Jan. 1992.

[31] M. Hajj-Hassan, V. P. Chodavarapu, and S. Musallam, "Microfabrication of ultra-long reinforced silicon neural electrodes," Micro Nano Lett., vol. 4, no. January, pp. 53-58, 2009.

\section{Biographies}

Alexandre Coumiotis Peixoto graduated, on 2008, in electrical Engineering, at University of Trás-osMontes e alto Douro, Vila Real, Portugal. He obtained in 2010 the Master's degree in Electrical Engineering, at the same institution. Presently, he is pursuing a PhD on Biomedical engineering at University of Minho, Guimarães, Portugal.

Sandra Beatriz Gonçalves graduated, on 2012, in Biomedical Engineering (Integrated Masters) with the major in Medical Electronics, at University of Minho, Portugal. Presently, she is a researcher at University of Minho. 
Francisco Pinho graduated, on 2002, in Physical Therapy and in industrial electronics engineering on 2009. Presently he is an assistant professor at Instituto Politécnico de Saúde do Norte (IPCA) and is also pursuing a PhD on biomedical engineering at University of Minho, Guimarães, Portugal.

Alexandre Ferreira da Silva graduated, in 2007, in Biomedical Engineering (Integrated Masters) with the specialization in Medical Electronics, at University of Minho, Braga, Portugal. He obtained in 2011 the $\mathrm{PhD}$ degree in Leaders for Technical Industries, at the same institution, over the MIT-Portugal Program within the Engineering Design and Advanced Manufacturing (EDAM) focus-area. Presently, he is an assistant professor to the MIT Portugal's EDAM focus-area at University of Minho.

Nuno Sérgio Dias graduated in industrial electronics and computers at University of Minho, Portugal in 2004. In 2009, he obtained the $\mathrm{PhD}$ degree in industrial electronics from the University of Minho, Guimarães, Portugal, in collaboration with the Center for Neural Engineering at The Pennsylvania State University, State College, PA, USA. During his PhD, Nuno developed a Brain-Machine Interface Based on Biotelemetry and Dry Electrodes. He is currently pursuing a post-doc position at ICVS/3Bs Associate Laboratory, University of Minho, Braga, Portugal.

José Higino Correia graduated in Physical Engineering from University of Coimbra, Portugal in 1990. $\mathrm{He}$ obtained in 1999 a $\mathrm{PhD}$ degree at the Laboratory for Electronic Instrumentation, Delft University of Technology, The Netherlands, working in the field of microsystems for optical spectral analysis. Presently, he is a Full Professor in Department of Industrial Electronics, University of Minho, Portugal. He was the General-Chairman of Eurosensors 2003 and MME 2007, Guimarães, Portugal. His professional interests are in micromachining and microfabrication technology for mixed-mode systems, solid-state integrated sensors, microactuators and microsystems. 